\title{
SUPPORTING PRIMARY CARE THROUGH PEDIATRIC TELEPSYCHIATRY
}

\author{
ANTONIO PIGNATIELLO \\ Telepsychiatry Program, Hospital for Sick Children and Division of Child Psychiatry, and \\ Department of Psychiatry, University of Toronto, Ontario \\ KATHERINE BOYDELL \\ Community Health Systems Resource Group, Child Health Evaluative Sciences Research \\ Institute, Hospital for Sick Children, and Department of Psychiatry and Dalla Lama School of \\ Public Health, University of Toronto, Ontario
}

JOHN TESHIMA

Department of Psychiatry, University of Toronto, and Sunnybrook Health Sciences Centre, Toronto, Ontario

TIZIANA VOLPE

Community Health Systems Resource Group, Hospital for Sick Children, and Institute of Medical Science, University of Toronto, Ontario

\begin{abstract}
This paper describes a pediatric telepsychiatry program serving communities in rural Ontario, Canada. Since the program's inception in 2000, over 4,300 direct clinical consultations have been provided for a variety of presenting issues. Indirect consultations and educational sessions have augmented clinicians' knowledge. Participant evaluation of the program indicates that a comprehensive model of psychiatric consultation and education can be delivered effectively via live interactive videoconferencing to support primary care and to enhance capacity in remote, underserviced areas in Ontario.
\end{abstract}

Almost $20 \%$ of children worldwide have one or more mental health disorders (Waddell \& Shepherd, 2002; World Health Organization, 2003). The prevalence of diagnosable mental health problems in children in the province of Ontario, Canada, is consistent with this worldwide rate, yet only 1 in 6 of these children receives mental health services (Offord et al., 1987). The ratio of child psychiatrists to children with mental health needs is approximately 1:6,148 (Steele \& Wolfe, 1999), while the estimated need for child psychiatrists is $14.3 / 100,000$ (or 1:1,390) children and youth (Thomas \& Holzer, 2006). Furthermore, although $18 \%$ of the province's population resides in rural Ontario, only $2 \%$ of child psychiatrists practice primarily in areas with populations of less than 20,000 (Steele \& Wolfe, 1999), and access to mental health services for children and adolescents is particularly limited in the 
vast, sparsely populated areas of northern Ontario. The provision of psychiatric services to children and their families in rural and remote regions must address geographic barriers to access (Boydell et al., 2006) as well as the distribution, attraction, and retention of psychiatrists and other mental health professionals.

Kirby \& Keon (2006) describe Canada's children's mental health system as fragmented and underfunded with a critical shortage of mental health professionals. They note that telepsychiatry has the potential to give underserviced regions access to existing specialized resources and to improve care, provided that a basic level of mental health service is already available in those communities. They recommend the use of telepsychiatry for consultations, education, and training of mental health professionals working in rural and remote areas.

Urness (2003, p. DP1) defines telepsychiatry as "the clinical use of live, interactive, full-motion, audiovisual communication, commonly referred to as videoconferencing." Telepsychiatry is efficient and likely cost-effective (Elford et al., 2000; Elford et al., 2001; Mair, Haycox, May, \& Williams, 2000; O'Reilly et al., 2007; Persaud et al., 2005); it is also user-friendly (Ermer, 1999). A population with diverse presenting problems and degrees of psychosocial severity can be managed (O'Reilly et al., 2007; Wooton, Yellowlees, \& McLaren, 2003), employing principles of community systems of care (Winters, Pumariega, Work Group on Community-Based Systems of Care, Committee on Community Child and Adolescent Psychiatry, \& Work Group on Quality Issues, 2007). A positive attitude (Werner, 2004), commitment, and flexibility are necessary attributes for promoting the viability of telemedicine programs (Hilty, Marks, Urness, Yellowlees, \& Nesbitt, 2004; Yellowlees, 2005). Telepsychiatry has also been used for a wide variety of educational purposes, including continuing education and supervision of medical trainees and mental health professionals (Hilty et al.). Collaboration among agencies and ministries serving the needs of children is key in creating community systems of care (Rygh \& Hjortdahl, 2007; Winters et al., 2007) and in realigning fragmented, parallel systems.

\section{THE TORONTO PEDIATRIC TELEPSYCHIATRY PROGRAM}

\section{Overview}

In 2000, the Ontario Ministry of Community and Social Services (now the Ministry of Children and Youth Services; MCYS) enlisted the Division of Child Psychiatry, University of Toronto, to create the Toronto Pediatric Telepsychiatry Program (TPTP). The program operates through the Hospital for Sick Children (SickKids) in Toronto, a 325-bed downtown teaching hospital with diverse psychiatric programs. SickKids is one of eight academic sites within the University of Toronto's Division of Child Psychiatry where clinical, teaching, and research mandates are followed.

The program provides bilingual (English and French) psychiatric consultations and education to underserviced children's mental health community agencies and their clients utilizing interactive videoconferencing (ITV). The program has also developed initiatives addressing specific needs, for example, services for Aboriginal communities and for young fire setters. 
Currently, the Ministry of Children and Youth Services has designated 15 primary ("far") sites, along with their satellite locations, ${ }^{1}$ to participate in the program. The sites are connected to the SickKids "hub" site via an Integrated Services Digital Network (ISDN) carried on a maximum of three lines (maximum bandwidth $384 \mathrm{kbits} / \mathrm{second}$ ), or via Internet Protocol (IP). The service is also accessible to any children's mental health agency in Ontario with compatible technology. The hub site is equipped with four studios: two housing two 36-inch CRT monitors each, one with a single 50-inch plasma monitor, and one containing a 17-inch LCD monitor. All configurations allow both hub and far sites to be viewed simultaneously. All cameras have local and remote pan-tilt-zoom control, and videoconferencing units (Polycom or Tandberg) enable the data transmission (see Figure 1).

\section{Figure 1}

Toronto Pediatric Telepsychiatry Program Videoconference

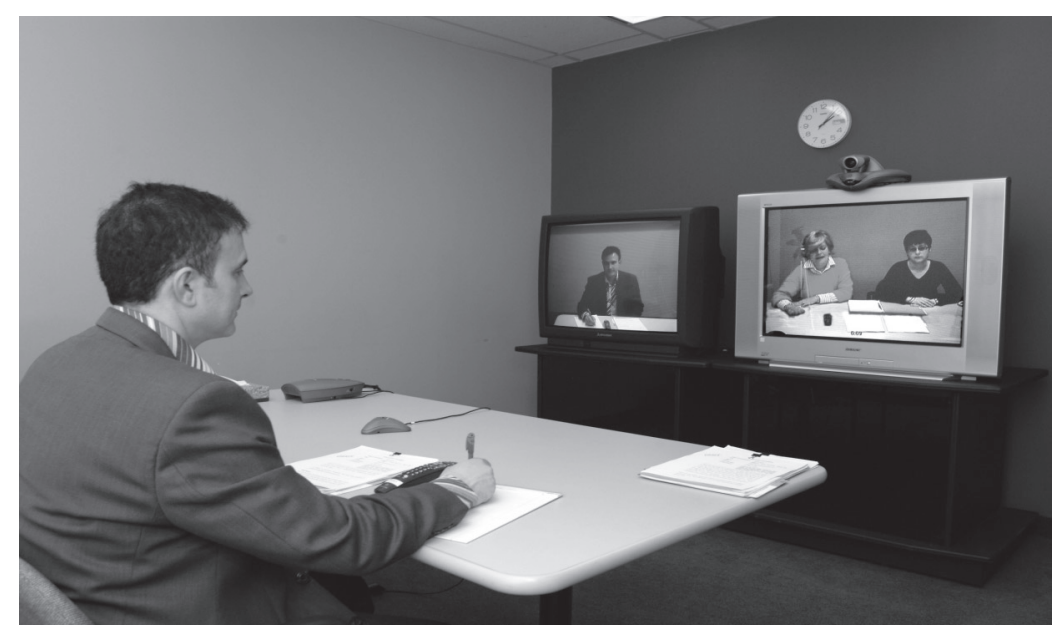

Seventy-five faculty (almost exclusively child psychiatrists) within the Division of Child Psychiatry at the University of Toronto are potentially available to provide consultations and other services. Of those, 14 participate on a regular weekly or monthly roster, providing the majority of the services, and an additional 16 faculty attend when requested for specific consultations or educational sessions, depending on their expertise and availability.

Core hub-site staff (Figure 2) and a designated telepsychiatry coordinator at each far site provide the necessary infrastructure. The hub-site staff meet on a monthly basis to discuss program-related issues and future planning. Steering Committee meetings are held quarterly with the core hub team and all sites via ITV, and minutes are recorded and circulated. Orientation of prospective and new sites is provided through ITV. 
Figure 2

Hub Site Organization

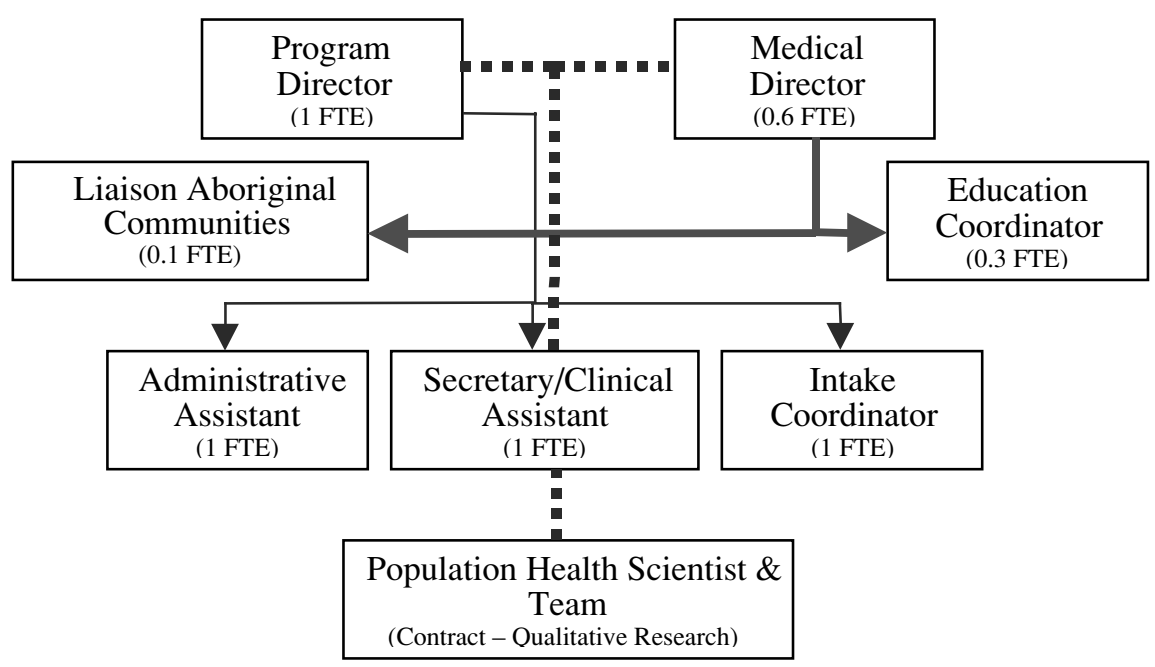

Ministry funding pays for capital and operating expenses and remuneration of clinical and administrative staff. Initial in-kind contributions from SickKids allowed for physical space and limited administrative support until 2007, when the Ministry of Children and Youth Services assumed full funding for the program.

\section{Services Provided}

Direct clinical consultation. As the TPTP is funded by the ministry responsible for children's mental health, referrals for direct clinical consultation are accepted from clinicians at community mental health agencies who act as overall case managers. Within the current mandate, referrals initiated by physicians are redirected through the local children's mental health agency, where available. Direct physician referrals are accommodated only under exceptional circumstances (i.e., there is no local children's mental health agency or there is a confidentiality or personal conflict between the client and the agency. Where physicians act as case managers, local agencies may facilitate the video connection and must adhere to all TPTP referral and attendance protocols.)

Agency clinicians are required to complete a mental health assessment prior to requesting the consultation. Written consent forms, developed in accordance with the Ontario Personal Health Information Protection Act (Ontario Hospital Association, Ontario Hospital e-Health Council, Ontario Medical Association, \& Office of the Information and Privacy Commissioner/Ontario, 2004), must be completed to confirm that the youth and/or family understand and consent to the provision of psychiatric consultation from the TPTP via an interactive video link. The consent form also allows for the exchange of relevant information, records, and reports between the referring agency and the TPTP, and 
for the release of a copy of the report to the treating physician who will be responsible for facilitating any further medical/pharmacological interventions. Participation of treating physicians is welcomed and encouraged through liaison with the agency case manager. Clients are made aware that information collected from the consultation will be entered into a database, in aggregate format, to be used for education, statistics, quality improvement, and other purposes permitted or required by law. If additional research is to be conducted, application is made to the SickKids Research Ethics Board, and specific consent forms are generated.

Supporting documentation and referrals are triaged by presented urgency and matched to compatible consultants. For non-urgent referrals, the average time from referral to consultation is approximately 3 to 5 weeks. The SickKids crisis team is available to provide urgent consultations within 24 to 48 hours. The child's case manager/primary clinician is required to be present during the consultation to bridge such issues as culture, language, formulation, and recommendations between client and consultant, and to confirm and clarify roles and responsibilities (Broder et al., 2002). Consultation is consultee-centred (Lambert, 2004), and the presence of the case manager and others involved in the care of the child or adolescent serves to expose health care providers to clinically and contextually relevant information, increasing knowledge and confidence. Impressions and recommendations are provided orally immediately after the consultation, and a written report follows within 15 working days. Follow-up consultations are not routinely provided as this is not a mandate of the program; however, they are available if necessary or requested. The TPTP maintains a database of standard referral forms and reports completed by all consultants. The reports list those present at the consultation and summarize diagnostic impressions, clinically assessed degree of psychosocial severity, and recommendations.

From May 1, 2000, to March 31, 2007, a total of 4,382 direct clinical consultations were provided (Figure 3): $22.5 \%$ were follow-ups, $1.1 \%$ were urgent, and $5.4 \%$ were in French. Of children and adolescents seen, $66.3 \%$ were male, and $19.6 \%$ were Aboriginal. Based on the psychiatric consultant's clinical impression of family, social, school and/or occupational function, and intensity of intervention recommended, the overall degree of dysfunction was mild in $16.4 \%$, moderate in $66.5 \%$, and severe in $17.1 \%$ of clients seen. Most frequent diagnoses based on clinical impressions are summarized in Table 1; the diagnostic categories are in keeping with other similar programs (Elford et al., 2000; Myers, Sulzbacher, \& Melzer, 2004). Actively psychotic children and youth have successfully participated in consultations. Intervention recommendations (e.g., medication, counselling, focused assessments, placement) are presented in Tables 2 and 3.

Indirect program consultation. Among the designated sites currently receiving services, 20 programs receive regular, monthly consultation. A consultant meets with a designated group of agency staff from the program (e.g., school-based day treatment programs, residential and foster homes, specialized programs working with children of military families) to discuss clinical, program-wide, and community issues. Upon registration in these identified agency programs, youth and family are asked to sign consent forms allowing issues related to the youth's management while in the program to be discussed in this indirect consultation modality. To be inclusive of local treating physicians, program staff also obtain the consent of the youth's physician to consult with a child psychiatrist about the 
patient. Following each consultation, the consultant summarizes in writing both the process and content of the discussion. Topics discussed have included individual youth and their behaviour; diagnosis, formulation, and management; program, system, and community issues; and transference and countertransference. At least annually, participants in the program consultation complete evaluations of the consultation process. An informal evaluation of this feedback suggests that rural and remote staff appreciate the education, support, and guidance in working with youth with complex problems and their families.

Figure 3

Direct Clinical Consultations 2000-2007 $(N=4,382)$

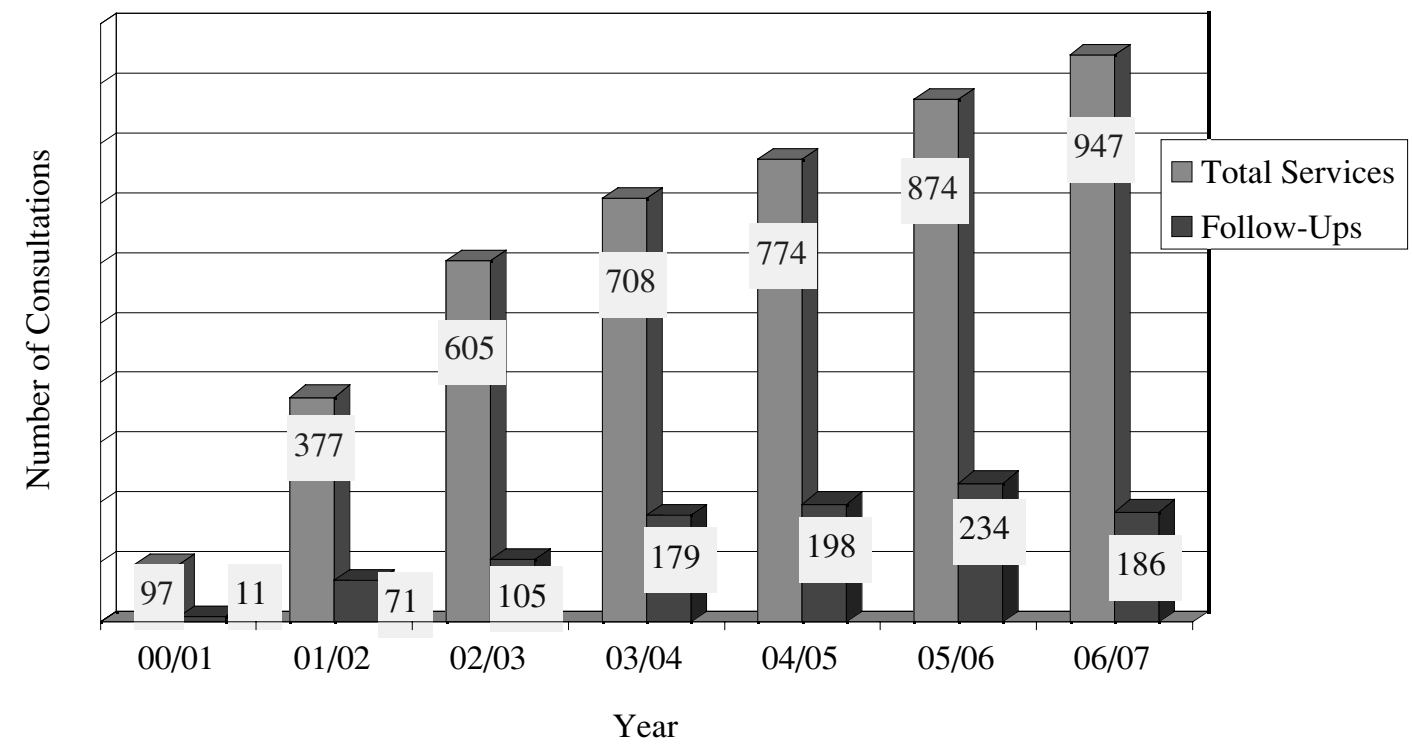

Table 1

Top 10 Diagnoses 2000-2007 ( $N=4,382$ Consultations)

Diagnosis

Attention deficit hyperactivity disorder

Mood disorders

Oppositional defiant disorder

Anxiety disorders

Learning disabilities

Relationship problems

Problems relating to abuse/neglect

Conduct disorder

Attachment disorder

Pervasive developmental disorder

\section{Total consultations (\%)}

47.2

29.3

20.7

19.8

17.4

17.1

11.9

11.0

8.1

6.8 
Table 2

Consultant Recommendations (Excluding Medications) 2000-2008

\begin{tabular}{lc}
\hline Recommendation & \% of total clients seen \\
\hline Further assessment & 16 \\
Psychological & 13 \\
Educational/academic & 3 \\
Speech/language & 6 \\
Medical/neurological & 53 \\
Therapy/counselling & 29 \\
Individual & 22 \\
Family & 13 \\
Parent & 4 \\
Psychoeducational & \\
Group & 22 \\
Other recommendations & 3 \\
School intervention & 5 \\
Application for support funding at school & 6 \\
Foster/out-of-home placement & 1 \\
Residential group home placement & \\
Day treatment & \\
\hline
\end{tabular}

Note. $N=4,906$ consultations, including follow-ups.

Table 3

Consultant Medication Recommendations 2000-2008

\begin{tabular}{lccc}
\hline Medication & Start (\%) & Discontinue (\%) & Change (\%) \\
\hline Stimulant & 13 & 1 & 8 \\
Antidepressant & 10 & 2 & 3 \\
Antipsychotic & 6 & 1 & 2 \\
Mood stabilizer & 2 & .4 & .7 \\
Anxiolytic & .8 & .2 & .2 \\
Other & .4 & .1 & .8 \\
\hline
\end{tabular}

Note. $N=4,906$ consultations, including follow-ups. No medication was recommended for $40 \%$ of the consultations; no medication change was recommended for $7 \%$ of the consultations.

Education. Exposing psychiatry residents at the hub site to telepsychiatry has been a particular priority of the TPTP in the hope that at least some residents would choose to participate in telepsychiatry in their future careers (Hodges, Rubin, Cooke, Parker, \& Adlaf, 2006). Since 2005, it has been mandatory for all University of Toronto psychiatry residents to attend two telepsychiatry consultations during their core 6-month rotation in child psychiatry. Between 2005 and 2007, 67 residents attended 115 
consultations and completed 101 evaluation forms. Eighty-seven percent (100/115) indicated that the experience was interesting and enjoyable, and $81 \%$ (93/115) indicated that they were more interested in participating in telepsychiatry in the future. An additional four residents have completed 3- or 6month electives in telepsychiatry. One of these residents subsequently established a practice in a northern Ontario community and has taken on a significant administrative role in the TPTP.

The continuing education program for community mental health professionals was developed on the basis of a detailed needs assessment consisting of a questionnaire completed by all the participating far sites, a subsequent ITV meeting to verify the questionnaire results, and informal feedback from the consultant psychiatrists working at the hub site. The continuing education program has been delivered in the format of multiple seminars in a series, using a range of interactive teaching methods (casebased problems, role plays, games, etc.). The program format and the use of a needs assessment were based on the evidence that these approaches are more likely to lead to changes in practice and to improved patient outcomes (Mazmanian \& Davis, 2002). In response to participants' requests, a calendar of educational events is prepared for 6-month blocks.

From 2000 to 2007, 97 sessions were presented on 22 different topics including anxiety disorders, depression, cognitive-behavioural therapy, behaviour management, and adolescent psychotherapy. Participants returned 6,058 evaluation forms, and a preliminary qualitative analysis found a number of emerging themes: (a) participants valued interactive techniques and the use of case examples, (b) the seminars reinforced existing knowledge, (c) the seminars provided new knowledge that could be applied to the participants' practices, (d) the seminars stimulated participants to reflect more on their clinical work, and (e) participants felt more confident about their practice.

Knowledge exchange. An interactive link via videoconference was established between the hospital's weekly psychiatry grand rounds and rural sites to allow physicians and other mental health professionals to benefit from the educational events at SickKids. To keep consultants apprised of relevant information in the TPTP, including dissemination of research findings, a biannual newsletter entitled Short Circuit is circulated electronically and on paper. The TPTP collaborates regularly with similar programs in other Canadian provinces as well as in the United States, England, and Australia to share initiatives, processes, protocols, and experiences. Additionally, national and international knowledge exchange occurs through mutual participation in psychiatric grand rounds on an annual basis.

\section{Evaluation}

Within the first year of the establishment of the Toronto Pediatric Telepsychiatry Program, an independent research team was engaged to develop and implement a comprehensive multiphase program of qualitative research. A critical review of the extant literature was conducted, and an annotated bibliography was produced. The review revealed little research on the educative and knowledge-transfer potential of telepsychiatry programs; most studies consisted of postencounter client satisfaction surveys that considered issues primarily of user comfort and satisfaction with the technology (Bishop, O’Reilly, Maddox, \& Hutchinson, 2002; Williams, May, \& Esmail, 2001). 
Input was obtained from all stakeholder groups (funder, hub site, and far site) through surveys, focus groups, and individual interviews. These groups identified the critical importance of considering all stakeholders in the evaluation and of taking into account the unique context (geographical, cultural, and social) of each telepsychiatry site and its community (Boydell, Greenberg, \& Volpe, 2004). Four studies have been completed or are underway.

Perspectives of service providers and family members. Service providers reported that having access to specialty mental health expertise through the TPTP enhanced their capacity to support clients (young people and their families) with specific and often complex problems. Increased confidence was also mentioned as a benefit; often, the telepsychiatry consultation served to confirm the diagnosis that the rural practitioner had already suspected and the appropriateness of the intervention. The program reduced the burden on family members, eliminating the need to drive long distances for specialty care, thus alleviating financial strain and decreasing time lost from work. Overall, family members and clinicians felt strongly supported through their consultation experience. Although faceto-face connection would be preferable, when that is not possible, consultation employing ITV was frequently cited as "the next best thing to being there" (Greenberg, Boydell, \& Volpe, 2006).

Perspectives of consulting psychiatrists, community general practitioners, and pediatricians. In face-to-face interviews, consulting psychiatrists reported that they valued their role as consultants as it meant that they were able to provide services without being burdened with an ongoing caseload. This helped with program buy-in and provided a robust directive to maintain a consultative model of service delivery. Research results also identified some difficulties encountered in the identification of non-verbal cues and subtle facial expressions during telepsychiatry consultations.

Physicians selected from a random sample of TPTP sites were interviewed via telephone. Many physicians were either unaware of the pediatric telepsychiatry program or lacked information about the ways that they could be involved; this is not necessarily surprising since TPTP services are currently directed at clinicians of children's mental health agencies. Physicians identified factors that would facilitate their involvement in the program, including remuneration issues and education regarding the prescribing of newer psychotropic medications (Boydell, Volpe, Watson-Gaze, Kertes, \& Greenberg, 2008). In response, the TPTP created medication information sheets and is developing a process for effective distribution.

Outcomes of consultation recommendations. One hundred telepsychiatry consultations were randomly selected from the TPTP program files, and data were reviewed for presenting issues, reasons for referral, and recommendations made during the consultation. It was found that multiple recommendations were made for youth with needs that were described as extremely complex. Successful implementation of recommendations was frequently the result of the value placed on the authority and expertise of consulting psychiatrists by case managers, family members, school personnel, and physicians. Additional factors that facilitated implementation were very specific treatment directives, meaningful engagement of the young person and his or her family, and the availability of follow-up sessions for complicated situations. Barriers to implementation included an absence of cooperation or willingness on the part of family members and youth, the brief nature of the consultation, and the scarcity of 
available community resources. The video technology and consultations by consultants unknown to the local community were not identified as barriers. Challenges were attributed to lack of local resources, treatment biases of families and young people, and high local staff turnover (Boydell, Volpe, Kertes, \& Greenberg, 2007).

Views of young people who have received a telepsychiatry consultation. A study funded by an external grant from the Provincial Centre of Excellence for Child and Youth Mental Health at the Children's Hospital of Eastern Ontario (CHEO) is currently in progress. Focus groups and individual interviews at two time points are being used to examine the experience of young people receiving telepsychiatry services.

\section{Lessons Learned}

Through relationship building and partnering with communities, telepsychiatry is well positioned to enhance-not replace-face-to-face delivery of health care, reduce professional isolation, and improve distribution of clinical expertise. Limitations imposed by catchment areas virtually disappear. Collaborative participation among caregivers and service providers representing multiple systems of care facilitates seamlessness and continuity as children, youth, and their families are readily triaged to appropriate services. Consultant recommendations lend extra weight in advocating for interventions that can be instituted locally (Boydell et al., 2007). Furthermore, care can remain within the local communities of children and their families, and services are available for communities that would otherwise be relatively isolated or where the population is too low to attract and retain on-site professional resources. In small, remote communities where clinicians and clients may have close knowledge or acquaintance of each other, receiving mental health services from a distant provider, via ITV, may offer a greater sense of anonymity, privacy, and confidentiality, which may reduce the stigma of receiving mental health intervention. The consultee-centred consultation model and the provision of continuing education, often to multiple sites simultaneously, contribute to the multiplier effect, transferring expertise from one to many. The practice of psychiatry, which largely employs history taking, observation, and collateral information gathering, lends itself well to the videoconferencing medium. This mode of service delivery can tackle challenges to access due to distance, terrain, climate, and traffic.

The consultation model itself, however, may pose challenges for those unfamiliar with it. The consultant is expected to provide a formulation and to make recommendations almost immediately. Community and agency cultural issues and provision of recommendations for services that are feasible and locally available require consideration.

Technologically, although image resolution is adequate, ITV does not allow for crisp detail of body language or facial affective nuances, and direct eye contact is lost (Tam, Cafazzo, Seto, Salenieks, \& Rossos, 2007). Additionally, alternative approaches must be found to confer empathic and social gestures (i.e., hand-shaking, sharing coffee, offering tissues). Communication via videoconferencing requires awareness of etiquette and extra consideration to adjust to the medium. It is important for participants to work with the technology rather than be frustrated by it. Institution of the electronic medical record could facilitate data transfer and sharing. 
Providing education to multiple sites simultaneously is very cost-effective. Depending on the number of participants, the delivery cost can be as low as $\$ 21.66 \mathrm{CDN}$ per attendant, per session. Although technological problems may occur, telepsychiatry is generally considered an appealing experience for trainees, and seminars can be delivered successfully via ITV to multiple and distant sites. However, time commitment and dedicated personnel are required to execute such a comprehensive educational program that is attentive to the needs of heterogeneous target audiences.

The Toronto Pediatric Telepsychiatry Program offers a comprehensive, critical initiative to address the shortage of child and adolescent mental health resources in rural Ontario, providing increased pediatric mental health knowledge and training to remote and underserviced areas of this province (Broder, Manson, Boydell, \& Teshima, 2004). Medical trainees in urban teaching centres are also expanding their knowledge of and comfort level with rural mental health issues, the consultation model, and the potential of videoconferencing to provide psychiatric services. Early intervention through this program for children and youth who are showing mental health symptomatology can reduce the strain on families, front-line workers, physicians, and community institutions, including schools, which may improve long-term outcomes.

\section{Future Directions}

Efforts are underway to expand the current mandate to include distant treating physicians more directly in the referral process and in the continuing education program, as this would emphasize a truly interprofessional approach to managing the mental health issues of children, youth, and their families.

A provincial expansion of the program to include 2 additional hub sites and 10 additional far sites is being developed. There are many models of service delivery (Nelson, Barnard, \& Cain, 2003; Winters et al., 2007; Wootton et al., 2003) in addition to the consultation model described that require further exploration. Ongoing national and international collaboration will advance our knowledge in the use of this new approach to providing mental health services to rural and remote communities.

\section{NOTE}

1. Big Trout Lake, Chatham, Fort Frances (satellite site: Atikokan), Kapuskasing (satellite sites: Hearst, Cochrane), Kenora, Moosonee, Owen Sound, Parry Sound, Pembroke (former site), Sault Ste. Marie, Sioux Lookout, Thunder Bay (satellite sites: Geralton/Longlac, Marathon, Nipigon), Kirkland Lake, Bracebridge (satellite site: Huntsville), North Bay (satellite site: Sundridge), and Timmins.

\section{RÉSUMÉ}

Cet article porte sur un programme de télépsychiatrie pédiatrique mis en place dans des communautés rurales de l'Ontario. Depuis ses débuts, en 2000, le programme a permis d'offrir plus de 4300 consultations cliniques directes en psychiatrie au cours desquelles différents problèmes ont été traités. Des consultations indirectes (c'est-à-dire par l'intermédiaire d'autres intervenants euxmêmes en contact avec des psychiatres) et des sessions de formation ont aussi permis d'accroître les compétences des intervenants installés dans la communauté. L'évaluation du programme que les par- 
ticipants ont faites montre que ce modèle intégré de consultations et de formation en psychiatrie, qui s'appuie sur des vidéoconférences, est efficace : il constitue une nette amélioration des services à l'étape des soins primaires, ce qui est important dans des régions éloignées, où les services se font souvent trop rares.

\section{REFERENCES}

Bishop, J.E., O'Reilly, R.L., Maddox, K., \& Hutchinson, L.J. (2002). Client satisfaction in a feasibility study comparing face-to-face interviews with telepsychiatry. Journal of Telemedicine and Telecare, 8, 217-221.

Boydell, K.M., Greenberg, N., \& Volpe, T. (2004). Designing a framework for the evaluation of paediatric telepsychiatry: A participatory approach. Journal of Telemedicine and Telecare, 10, 165-169.

Boydell, K.M., Pong, R., Volpe, T., Tilleczek, K., Wilson, E., \& Lemieux, S. (2006). Family perspectives on pathways to mental health care for children and youth in rural communities. Journal of Rural Health, 22, 182-188.

Boydell, K.M., Volpe, T., Kertes, A., \& Greenberg, N. (2007). A review of the outcomes of the recommendations made during paediatric telepsychiatry consultations. Journal of Telemedicine and Telecare, 13, 277-281.

Boydell, K.M., Volpe, T., Watson-Gaze, J., Kertes, A., \& Greenberg, N. (2008). Physicians weigh in: Medical perspectives on a paediatric telepsychiatry program in Ontario. Unpublished manuscript.

Broder, E., Cheng, A., Cooke, R., Hodges, B., Ghandi, J., McCabe, L., et al. (2002). Telepsychiatry guidelines and procedures for clinical activities. Toronto, ON: University of Toronto Psychiatric Outreach Program.

Broder, E., Manson, E., Boydell, K., Teshima, J. (2004). Use of telepsychiatry for child psychiatric issues: First 500 cases. CPA Bulletin, 36(3), 11-15.

Elford, R., White, H., Bowering, R., Ghandi, A., Maddiggan, B., St. John, K., et al. (2000). A randomized, controlled trial of child psychiatric assessments conducted using videoconferencing. Journal of Telemedicine and Telecare, 6, 73-82.

Elford, D.R., White, H., St. John, K., Maddigan, B., Ghandi, M., \& Bowering, R. (2001). A prospective satisfaction study and cost analysis of a pilot child telepsychiatry service in Newfoundland. Journal of Telemedicine and Telecare, 7, 73-81.

Ermer, D.J. (1999). Experience with a rural telepsychiatry clinic for children and adolescents. Psychiatric Services, 50, 260-261.

Greenberg, N., Boydell, K.M., \& Volpe, T. (2006). Pediatric telepsychiatry in Ontario: Caregiver and service provider perspectives. Journal of Behavioral Health Services \& Research, 33, 105-111.

Hilty, D.M., Marks, S.L., Urness, D., Yellowlees, P.M., \& Nesbitt, T. (2004). Clinical and educational telepsychiatry applications: A review. Canadian Journal of Psychiatry, 49, 12-23.

Hodges, B., Rubin, A., Cooke, R.G., Parker, S., \& Adlaf, E. (2006). Factors predicting practice location and outreach consultation among University of Toronto psychiatry graduates. Canadian Journal of Psychiatry, $51,218-225$.

Kirby, M.J.L., \& Keon, W.J. (2006). Out of the shadows at last: Transforming mental health, mental illness and addiction services in Canada. Highlights and Recommendations: Children and Youth (pp. 135-155). Ottawa: Standing Senate Committee on Social Affairs, Science and Technology. Retrieved August 18, 2008, from http://www.parl.gc.ca/39/1/parlbus/commbus/senate/com-e/soci-e/rep-e/rep02may06part2e.htm\#_Toc133223085

Lambert, N.M. (2004). Consultee-centered consultation: An international perspective on goals, process, and theory. In N.M. Lambert, J.H. Sandoval, \& I. Hylander (Eds.), Consultee-centered consultation: Improving the quality of professional services in schools and community organizations (pp. 3-19). Mahwah, NJ: L. Erlbaum Associates.

Mair, F.S., Haycox, A., May, C., \& Williams, T. (2000). A review of telemedicine cost-effectiveness studies. Journal of Telemedicine and Telecare, 6(Suppl. 1), S38-S40.

Mazmanian, P.E., \& Davis, D.A. (2002). Continuing medical education and the physician as a learner: Guide to the evidence. Journal of the American Medical Association, 288, 1057-1060. 


\section{SUPPORTING PRIMARY CARE THROUGH PEDIATRIC TELEPSYCHIATRY}

Myers, K.M., Sulzbacher, S., \& Melzer, S.M. (2004). Telepsychiatry with children and adolescents: Are patients comparable to those evaluated in usual outpatient care? Telemedicine Journal and e-Health, 10, 278-285.

Nelson, E.-L., Barnard, M., \& Cain, S. (2003). Treating childhood depression over videoconferencing. Telemedicine Journal and e-Health, 9, 49-55.

Offord, D.R., Boyle, M.H., Szatmari, P., Rae-Grant, N.I., Links, P.S., Cadman, D.T., et al. (1987). Ontario Child Health Study II. Six-month prevalence of disorder and rates of service utilization. Archives of General Psychiatry, 44, 832-836.

Ontario Hospital Association, Ontario Hospital e-Health Council, Ontario Medical Association, \& Office of the Information and Privacy Commissioner/Ontario. (2004). Physician privacy toolkit: Guide to the Ontario Personal Health Information Protection Act. Toronto: Queen's Printer for Ontario. Retrieved August 18, 2008, from http://www.oma.org/phealth/privacy/physiciankit_FINAL.DOC

O’Reilly, R., Bishop, J., Maddox, K., Hutchinson, L., Fisman, M., \& Takhar, J. (2007). Is telepsychiatry equivalent to face-to-face psychiatry? Results from a randomized controlled equivalence trial. Psychiatric Services, $58,836-843$.

Persaud, D.D., Jreige, S., Skedgel, C., Finley, J., Sargeant, J., \& Hanlon, N. (2005). An incremental cost analysis of telehealth in Nova Scotia from a societal perspective. Journal of Telemedicine and Telecare, 11, 77-84.

Rygh, E.M., \& Hjortdahl, P. (2007). Continuous and integrated health care services in rural areas. A literature study. Rural and Remote Health, 7, Article 766. Retrieved August 18, 2008, from http://www.rrh.org.au/ publishedarticles/article_print_766.pdf

Steele, M.M., \& Wolfe, V.V. (1999). Child psychiatry practice patterns in Ontario. Canadian Journal of Psychiatry, 44, 788-792.

Tam, T., Cafazzo, J.A., Seto, E., Salenieks, M.E., \& Rossos, P.G. (2007). Perception of eye contact in video teleconsultation. Journal of Telemedicine and Telecare, 13, 35-39.

Thomas, C.R., \& Holzer, C.E., III. (2006). The continuing shortage of child and adolescent psychiatrists. Journal of the American Academy of Child and Adolescent Psychiatry, 45, 1023-1031.

Urness, D. (2003). Telepsychiatry (Discussion Paper 1). Canadian Psychiatric Association Bulletin, 35(3) [outsert]. Retrieved August 18, 2008, from http://publications.cpa-apc.org/media.php?mid=151

Waddell, C., \& Shepherd, C. (2002). Prevalence of mental disorders in children and youth. Vancouver: University of British Columbia, Mental Health Evaluation and Community Consultation Unit. Retrieved August 18, 2008, from http://www.mcf.gov.bc.ca/mental_health/mh_publications/02a_cymh.pdf

Werner, P. (2004). Willingness to use telemedicine for psychiatric care. Telemedicine Journal and e-Health, 10, 286-293.

Williams, T.L., May, C.R., \& Esmail, A. (2001). Limitations of patient satisfaction studies in telehealthcare: A systematic review of the literature. Telemedicine Journal and E-Health, 7, 293-316.

Winters, N.C., Pumariega, A., Work Group on Community-Based Systems of Care, Committee on Community Child and Adolescent Psychiatry, \& Work Group on Quality Issues. (2007). Practice parameter on child and adolescent mental health care in community systems of care. Journal of the American Academy of Child and Adolescent Psychiatry, 46, 284-299.

Wootton, R., Yellowlees, P., \& McLaren, P. (Eds.). (2003). Telepsychiatry and e-mental health. London: Royal Society of Medicine Press.

World Health Organization. (2003). Caring for children and adolescents with mental disorders: Setting WHO directions. Geneva: Author. Retrieved August 18, 2008, from http://www.who.int/mental_health/media/en/ 785.pdf

Yellowlees, P.M. (2005). Successfully developing a telemedicine system. Journal of Telemedicine and Telecare, 11, 331-335. 